\title{
Probabilistic rotor life assessment using reduced order models
}

\author{
Brian K. Beachkofski* \\ Air Force Research Laboratory, Propulsion Directorate, 1950 5th Street, Wright-Patterson AFB, OH 45433, USA
}

Received 25 October 2007

Revised 12 November 2008

\begin{abstract}
Probabilistic failure assessments for integrally bladed disks are system reliability problems where a failure in at least one blade constitutes a rotor system failure. Turbine engine fan and compressor blade life is dominated by High Cycle Fatigue (HCF) initiated either by pure HCF or Foreign Object Damage (FOD). To date performing an HCF life assessment for the entire rotor system has been too costly in analysis time to be practical. Although the substantial run-time has previously precluded a full-rotor probabilistic analysis, reduced order models make this process tractable as demonstrated in this work. The system model includes frequency prediction, modal stress variation, mistuning amplification, FOD effect, and random material capability. The model has many random variables which are most easily handled through simple random sampling.
\end{abstract}

Keywords: High cycle fatigue, probabilistic analysis, reduced order model, system reliability

\section{Introduction}

Traditional fan and compressor High Cycle Fatigue (HCF) design assumes a tuned rotor. The analysis uses single-blade vibration expanded to the full rotor by using cyclic symmetry [7]. This paper combines reduced order models for a complete rotor so that a probabilistic HCF reliability assessment can be accomplished on a rotor basis instead of a single blade model. As rotors migrate from inserted blades to integrally bladed rotors (IBR) or blisks, the system analysis becomes more important. Blades on an IBR or blisk are not replaceable since the blades and disk are a single component and there is no approved weld repair for replacing blades.

The primary defense against HCF is avoiding low order excitation of vibratory modes as seen on a Campbell diagram [15]. The concept is that, although you cannot avoid the excitation, accelerating or decelerating through resonance does not accumulate enough damaging cycles to fail a blade. Avoiding operation with the excitation frequency at the response frequency is the primary method of defending against HCF.

Another tool to design against HCF failures is forced response analysis. The steady and alternating stresses are compared to the material's Goodman diagram [7]. The allowable design stress is set at $60 \%$ of the material capability of the Goodman diagram as a design practice [1]. Stress magnitude can be estimated through modal force calculations and aerodynamic damping measures. If there is insufficient margin on the Goodman diagram, residual compressive stress can be applied through techniques such as Low-Plasticity Burnishing [14] or Laser Shock Peening [6] to the regions of likely crack propagation.

There are significant problems with the assumptions behind that design process. First, it is well demonstrated that small manufacturing variations in each blade create a mistuned rotor in which the alternating stress may be much higher than a single blade analysis predicts [3].

\footnotetext{
*Corresponding author. Tel.: +1 937255 7219; E-mail: brian.beachkofski@wpafb.af.mil.
} 


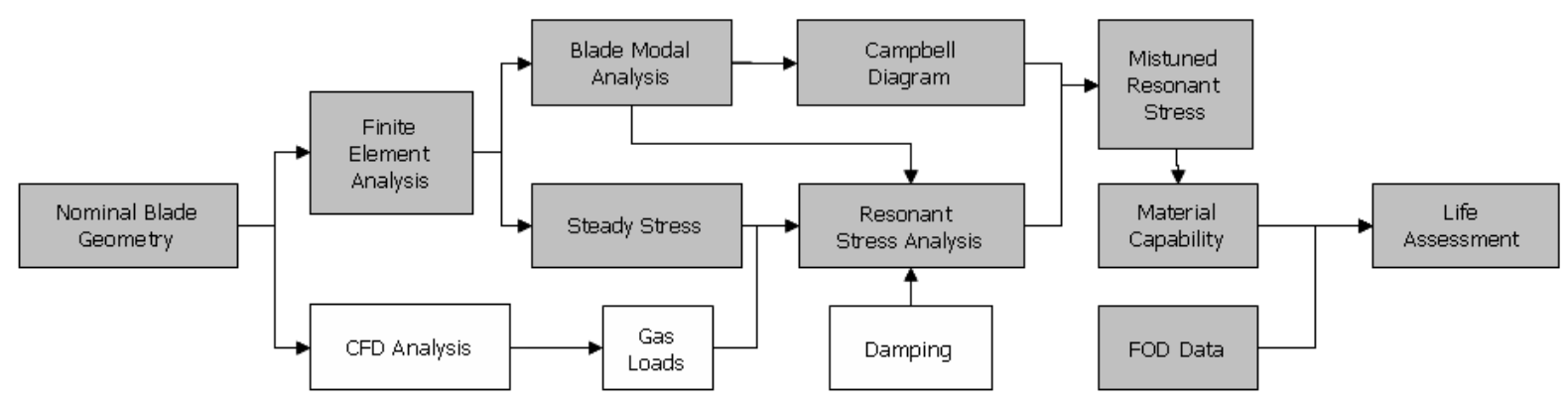

Fig. 1. Probabilistic HCF design process.

The second issue is that although there is a reliability metric to which the blades are designed, the blade is designed to be tolerant of low probability events like severe FOD at all points [1]. The result of the assumption is that the design is overly conservative, being designed to be tolerant of highly damaging events even where such damage is not likely to occur. For example, if the failure mode has a likelihood much lower that reliability requirement, there may not be a need to make the design tolerant of that damage.

When pricing the maintenance and part forecast for inserted blades, the impact may not be very significant. However, if the same assumptions are used in integrally bladed rotor analysis, the cost of supporting the system may be different than predicted, since cost of a complete rotor is much greater than a single blade.

This paper shows the viability of a rotor-based HCF life analysis rather than a blade-alone analysis with mistuned amplification factors. The analysis follows the HCF design process developed in the National HCF Initiative and accepted by turbine engine manufacturers and reported in the Engine Structural Integrity Program (ENSIP) Handbook [1]. While each of the reduced order models have been used independently, there is gap in the literature of a complete $\mathrm{HCF}$ analysis linking these new tools together to determine the practicality of an integrated probabilistic $\mathrm{HCF}$ rotor reliability analysis.

\section{System model}

During the National HCF Initiative [16] the government and industry team developed a process for HCF probabilistic design. The elements and process flow are shown in Fig. 1. The analysis in this paper includes the boxes that are highlighted in gray. Reduced order Computational Fluid Dynamics (CFD) is being actively pursued, but is still unreliable as of today. The damping values are also invariant since the dominant damping in an Integrally Bladed Rotor (IBR) is aerodynamic damping and also depends on CFD predictions.

The IBR analyzed in this system is a 24 blade system, of which 13 blades were measured using a coordinate measurement machine (CMM). The CMM data feeds the geometry variation model to generalize the off-nominal dimensions. The off-nominal geometry is evaluated in a Finite Element Analysis to produce the alternating stress. These amplitudes are increased through mistuning. The magnitudes of the stresses are compared to material capability through a random fatigue limit. The material capability is degraded by Foreign Object Damage induced stress concentration if the simulation shows FOD occurred.

The elements that are not included in this analysis are the CFD and damping. Variation in aerodynamic loading and its effect on structural response has been recognized and evaluated for some time [4]. Still, the lack of numerically efficient transonic CFD or linear sensitivity to geometric variation continues to hamper usage for analyses such as this one. As a result, a nominal forced response magnitude is assumed for the analysis.

There are two major components of damping in HCF analysis: aerodynamic and friction. Additionally, there is inherent material damping which is so small compared to the other two that it is generally considered insignificant.

Friction damping, significant enough in traditional fan and compressor rotors to cause mistuned response [10] or change mistuned response [13], does not factor in IBR analysis where the dovetail slot/post interface does not exist. While not including friction damping is obvious, the exclusion of aerodynamic damping is limited for the same reason that CFD is not included. To calculate the aerodynamic damping, the pressure profile on the blade is 


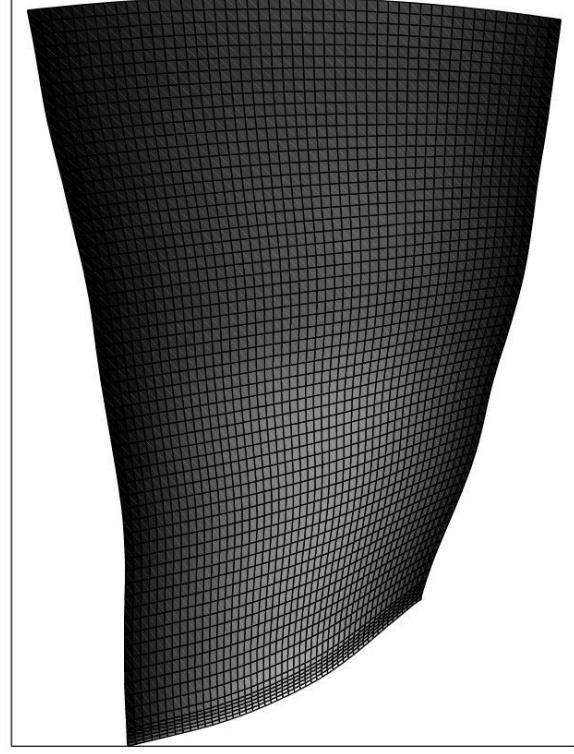

(a) Average Blade
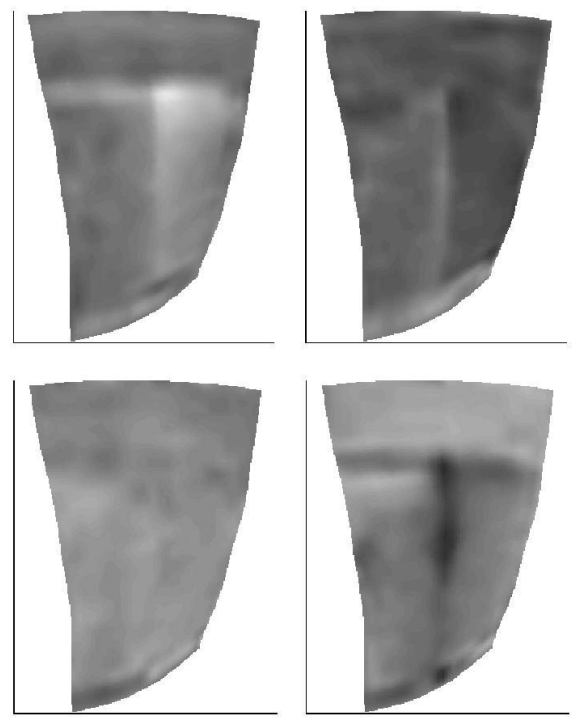

(b) Example Thickness Variations

Fig. 2. Blade dimension measurements.

integrated with motion of the blade to determine the sum of the pressure. Without CFD analysis, there is no way to calculate the aerodynamic damping and a nominal damping value is assumed as part of the nominal forced response magnitude.

Even excluding the CFD derived variations, the purpose of the analysis is still valid. The system is intended to show that multiple individual reduced order models can be linked through a probabilistic integration method. Those aspects that are included are described in detail in the following subsections.

\subsection{Geometry model}

As manufactured, blades do not match the design geometry exactly due to tolerances and variation in fabrication processes. A Coordinate Measurement Machine (CMM) is used as a quality control check at the end where the dimensions of the blades are compared to the design to ensure they are within design tolerances. The CMM uses either a laser probe for the blade surface or a stinger probe for the edges where reflection makes the laser measurements unreliable. CMM results from a manufactured rotor are the basis of the geometry variability in this work.

The average of thirteen blades' measurements is shown in Fig. 2 along with the examples of the deviation from average for specific blades. The deviations are used in a Principal Component Analysis (PCA) to reduce the number of random variables from each of the nodes on the blade surface to a much smaller count [2].

PCA transforms the basis for the thickness vector $(\underline{x})$ from from the individual nodes to a set of orthogonal vectors called the PCA modes. The PCA modes are the eigenvectors $(\psi)$ of the nodal thicknesses' covariance matrix. Each deviation can then be expressed as the mean thickness $\left(\mu_{\underline{x}}\right)$ plus a linear combination of the PCA modes, as shown in 1 , with the participation $(a)$ of each PCA mode $(j)$ in blade realization $(i)$. Three typical modes are shown in Fig. 3.

$$
\underline{x}_{i}=\mu_{\underline{x}}+\sum_{j=1}^{n} a_{i, j} \psi_{j}
$$

The vectors associated with the lower eigenvalues account for more of the variation than the higher number modes. The amount of variability that is accounted for in each PCA mode is shown in a Pareto chart such as Fig. 4. The cumulative variability included is also shown in the chart. The thicknesses can be approximated by using a subset of 

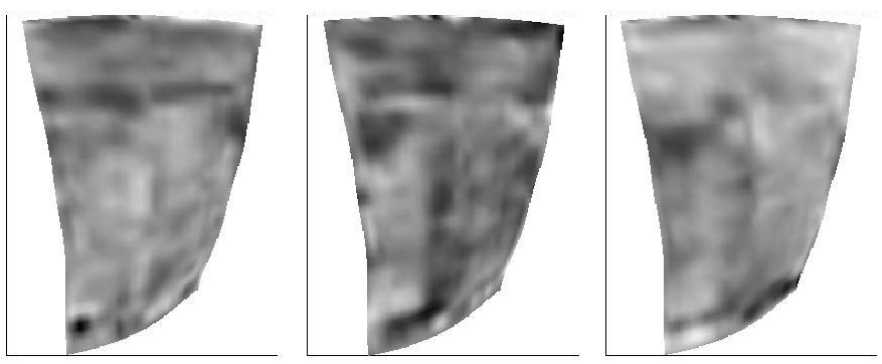

Fig. 3. Example PCA modes.

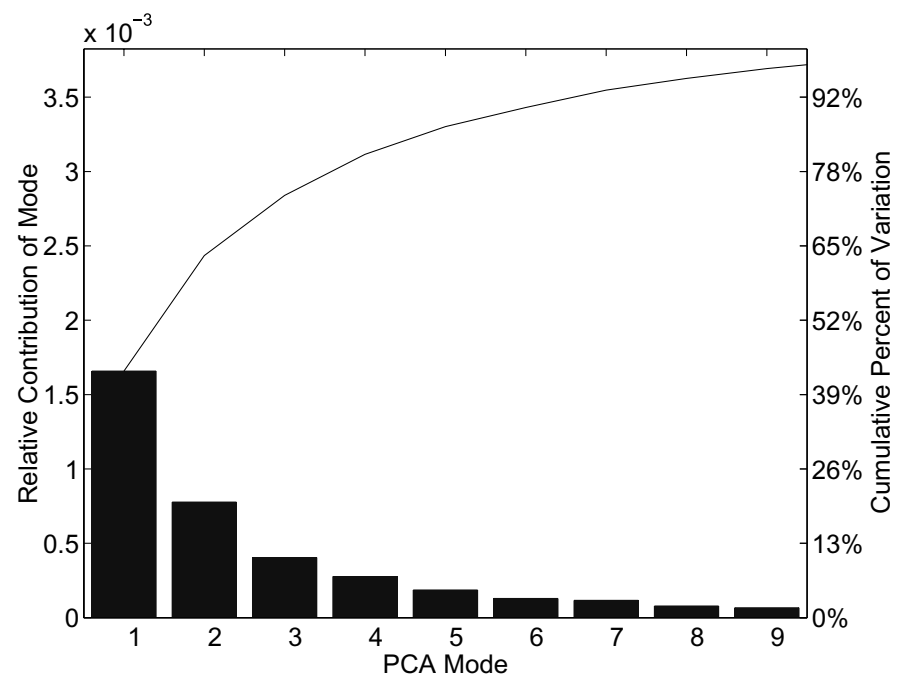

Fig. 4. Pareto chart of PCA modes.

the total number of modes. For example, if the modes are truncated at 8 rather than including all 13, the geometries that are recreated from the PCA modes will only account for $93 \%$ of the blade-to-blade variability. This analysis uses an 8 mode truncation for generating random blades.

$$
\underline{x}_{i} \approx \mu_{\underline{x}}+\sum_{j=1}^{m} a_{i, j} \psi_{j} \text { where } m<n
$$

When generating random, typical thickness distributions, the modal participation coefficients $(a)$ are randomly generated from Gaussian distributions. The distributions' means are zero since the mean data was extracted. The standard deviations for each mode are equal to those of the $13 \mathrm{CMM}$ data sets. Since the distribution of the generated population match the original data, the generated blades are likely estimates of blades made from the same manufacturing process.

\subsection{Modal analysis}

The modal response is determined by using Finite Element Analysis (FEA). The blades are modeled with plate elements, 50 chord-wise and 64 radially. However, rather than rebuild a Finite Element Model (FEM) for each random realization of blade geometry, the sensitivity of the nominal blade's frequency to each PCA mode predicts the off nominal geometry natural frequency. These sensitivities create a first order response surface to model, Eq. (3), the natural frequency that directly map the participation factors to a frequency. 


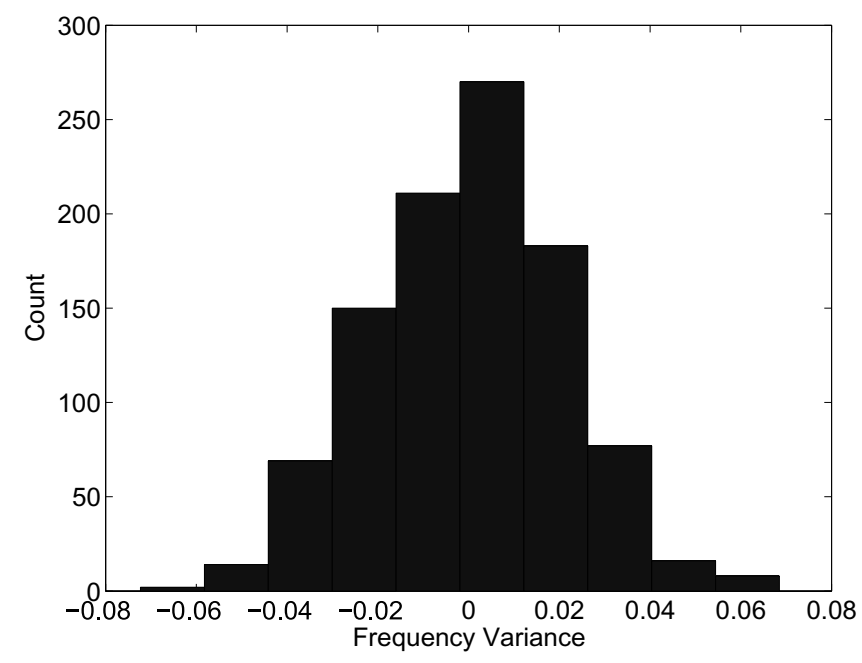

Fig. 5. Histogram of 1000 random blade frequencies.

$$
\lambda \approx \sum_{i=1}^{m} a_{i} \frac{\partial \lambda}{\partial \psi_{i}}
$$

The sensitivities can either be found using finite differencing or the less computationally demanding semi-analytical methods. Using this method, the mass and stiffness matrices only need to be formed, not solved, saving the majority of the time. Given an undamped system with harmonic motion where the mode shapes have been mass normalized, the eigenvalue sensitivity is as shown in Eq. (4).

$$
\frac{\partial \lambda_{i}}{\partial \psi_{j}}=\phi_{i}{ }^{T}\left(\frac{\partial K}{\partial \psi_{j}}-\lambda_{i} \frac{\partial M}{\partial \psi_{j}}\right) \phi_{i}
$$

Brown et al. [2] showed that the linear model for predicting natural frequencies is very accurate at least to the tenth mode of vibration. Continued unpublished work since then shows the linear model accurately predicts vibratory frequencies up to the twentieth mode. This system represents a fundamental mode where sensitivity analysis performs extremely well. Figure 5 shows the normalized frequency variation of 1,000 randomly generated blades.

The other aspect of the modal analysis is the alternating stress. This analysis assumes that the leading edge of the blade is always the crack initiation site so only the stresses at those nodes are tracked. Another simplifying assumptions is that a subset (ten) of those nodes will as accurately represent the stress case as the complete set. At a node the stress sensitivity to the PCA modes determines the stress along the leading edge paralleling the approach in the frequency analysis, meaning $j \in[1 \ldots 8]$ in Eq. (5).

$$
\sigma_{j} \approx \sum_{i=1}^{m} a_{i} \frac{\partial \sigma_{j}}{\partial \psi_{i}}
$$

The semi-analytical sensitivity is based on the mode shape sensitivities. The mode shape sensitivity formulation is:

$$
\frac{\partial \phi_{j}}{\partial \psi}=\sum_{k} c_{j k} \phi_{k}
$$

when $j \neq k$ :

$$
c_{j k}=\frac{\phi_{k}^{T}\left(\frac{\partial K}{\partial \psi}-\lambda_{k} \frac{\partial M}{\partial \psi}\right) \phi_{j}}{\lambda_{j}-\lambda_{k}}
$$

when $j=k$ : 


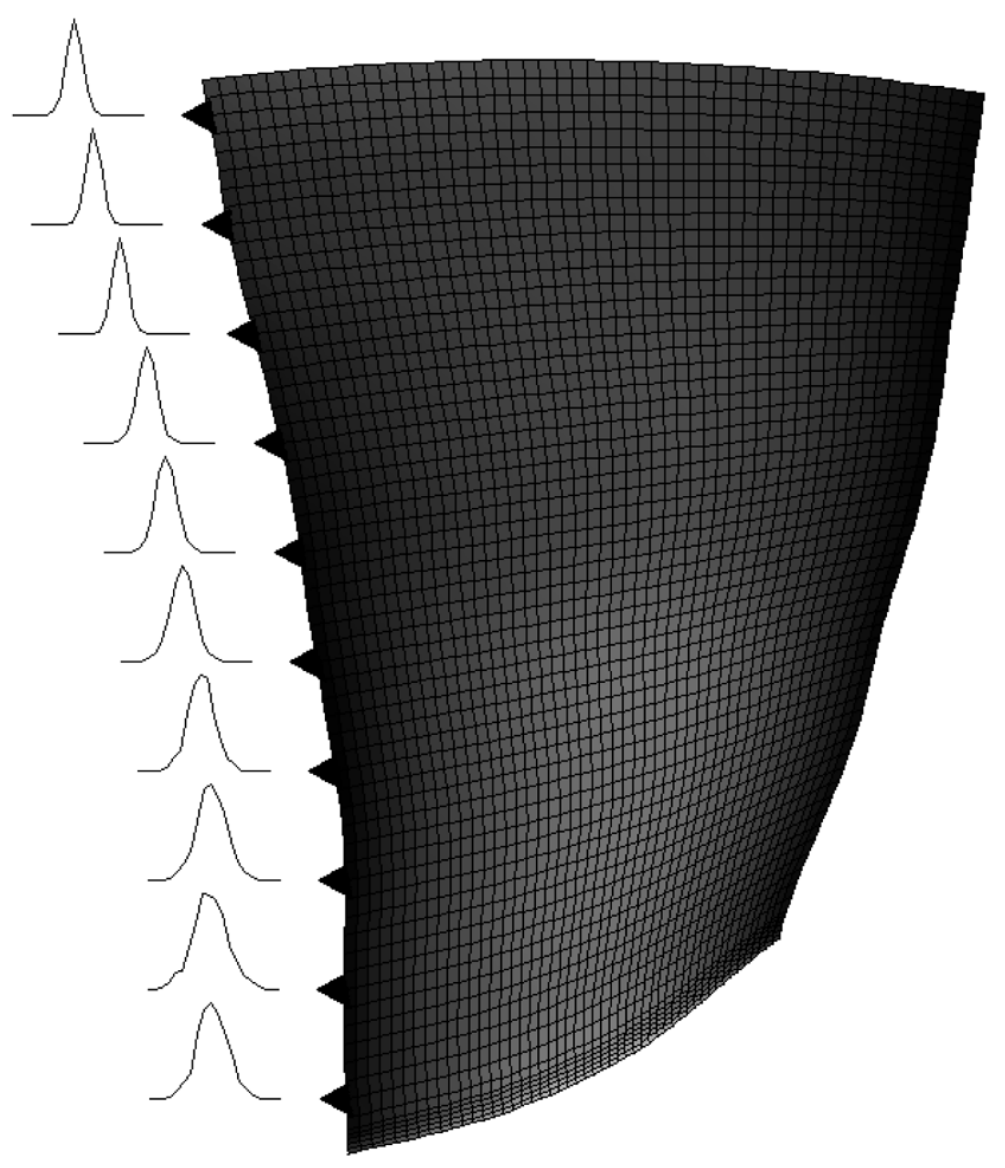

Fig. 6. Stress distributions at leading edge nodes.

$$
c_{j j}=-\frac{1}{2} \phi_{j}^{T} \frac{\partial M}{\partial \psi} \phi_{j}
$$

The Finite Element Analysis (FEA) software converts the mode shape to the stress map through the straindisplacement matrix, $B$. The mode shape, a set of displacements, is multiplied by $B$ to get the strain which are multiplied by the constitutive laws embodied in the $C$ matrix to calculate the stress. The sensitivities with respect to PCA modes are calculated in Eq. (9). The constitutive laws do not change with respect to changes in the geometry. The sensitivity of $B$ to geometry changes $(\psi)$ is due to the Jacobian matrix relating the actual geometry to standard element space.

$$
\frac{\partial \sigma}{\partial \psi}=C \cdot B \cdot \frac{\partial \phi_{i}}{\partial \psi}+C \cdot \frac{\partial B}{\partial \psi} \cdot \phi_{i}
$$

The distribution of stresses changes along the leading edge because of the varying sensitivities. Figure 6 shows the stress distribution at the 10 leading edge locations for 1,000 randomly generated blades. One thing to note is that the variance in stress is greater near the root than at the tip.

\subsection{Mistuning model (FMM)}

Mistuning is the when a nominally tuned system has small variations in the natural frequencies of blades on a rotor when it is manufactured. Mistuning has been demonstrated to cause large response variation, including amplification of forced response magnitude [8]. Traditional mistuning models assume the nominal mode shape remains unchanged and depend only on the blade frequency variation. Still, the mode shape was used to determine 


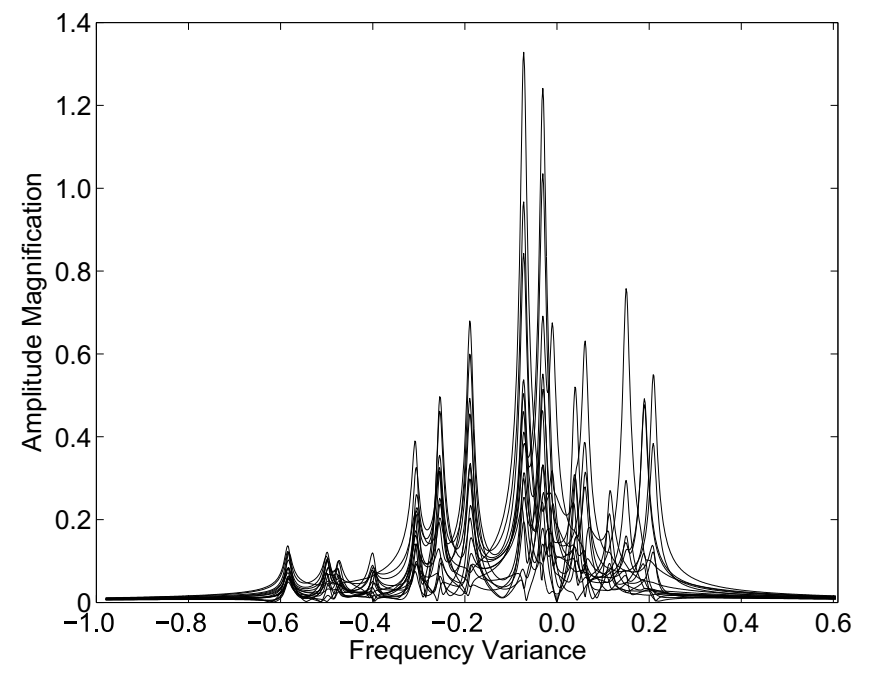

Fig. 7. Normalized mistuned blisk FRF.

the coupling from one blade to another. Recently, Feiner and Griffin [9] developed a simplified mistuning prediction model that works well for low order modes with separated mode families, the Fundamental Mistuning Model (FMM). The only information that the model needs are the tuned system frequencies and the mistuned blade frequencies from the frequency analysis.

FMM translates that information into a displacement amplification factor for each blade. The same assumptions that FMM uses, low-order mode widely spaced from other modal families, mean that the stress-displacement relationship is linear for small deflections. The calculated displacement amplification is therefore the same as the stress amplification. Figure 7 shows the normalized Forced Response Function (FRF) of each blade. Many amplification factors are actually attenuation factors since they are less than the tuned response as showed by a peak amplitude magnifications factor less than 1.0.

For the purposes of this analysis it is assumed that during engine operation the entire family of modes is exposed to vibration so that the maximum peak for each blade represents the stress amplification. Since the entire family of frequencies is designed away from steady state operating conditions, there is no particular frequency that has any greater chance of exposure than another. The amplification factor is multiplied with the leading edge stresses to the corresponding blade to determine the final alternating stresses in the blade.

\subsection{Foreign object damage}

A major factor in the stress at the leading edge is the presence of FOD [5]. Foreign Object Damage occurs when the engine ingests some material that strikes engine components. Usually this results in either a tear, dent, or gouge, all of which increase the local stress above the smooth shape [12]. The extent of increase in stress is shape dependent and measured by a stress intensity factor $\left(K_{f}\right)$. The magnitude of $K_{f}$ is dependent on the energy and angle of impact as well as the material of both the foreign object and the blade.

As a blade rotates about the axis, a point farther out radially sweeps a line that is longer than one closer to the axis. If debris is uniformly distributed in the inlet, the probability of a point being hit increases as the point is further out radially. This model acknowledges that fact by keeping the probability of FOD proportional to the area that portion of the blade sweeps.

When FOD does occur at a node, the process is very chaotic and predicting the FOD to model it is near impossible. Because of this, HCF life assessment does not use physic-based impact models; there is only a statistical model that matches the distribution of the damage. The model used in this case is a log-normal distribution with parameters 0.9 and 0.2. There are 10 binomial statistics per blade determining whether damage occurs and another 10 determining the stress intensity factor if FOD does occur. 


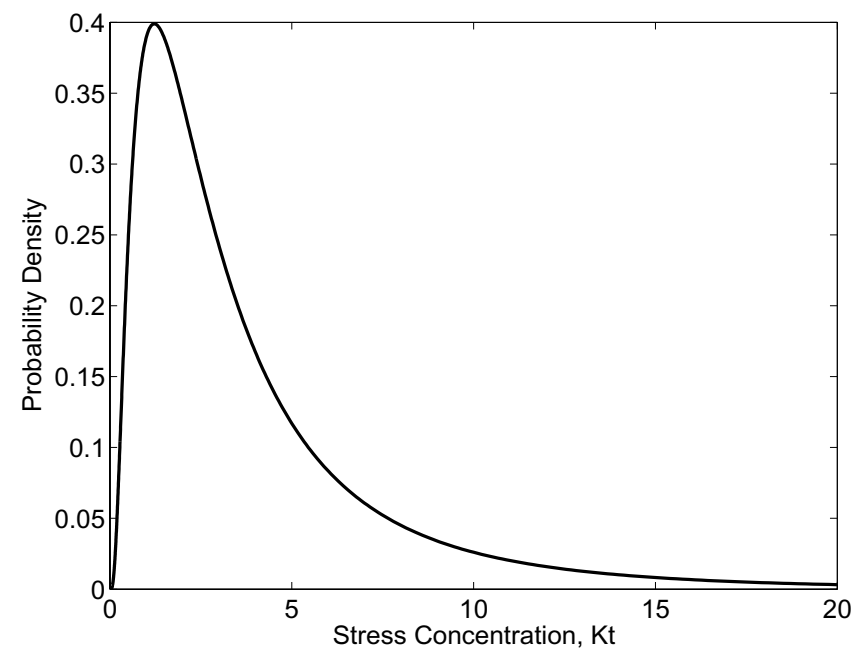

Fig. 8. Foreign object damage distribution.

The final stress value is tracked for each node and consists of the modal stress, a FOD $K_{t}$ if applicable, and the mistuning amplification. These contributors multiply together to quantify the stress demand at different locations on the blade.

\subsection{Material capacity}

The Random Fatigue Limit (RFL) model developed by [11] is a stochastic model of material capability. This model determines whether the stress demand at any node exceeds the blade capability. While each blade does have slightly different capability, there is a high correlation since they are all from the same rotor ingot. This analysis assumes that $10^{6}$ cycles are seen before the blade is inspected, so if the stress exceeds capability the crack grows and the rotor fails.

Equation (10) shows the CDF values $(F)$ for a given stress level $(x)$ of the cycles to failure $(w)$. The CDF value of a standard normal statistic is $\Phi(\cdot)$ and the Probability Density Function (PDF) of a normal statistic is $\phi(\cdot)$.

$$
F=\int_{-\infty}^{x} \frac{1}{\sigma_{\gamma}} \Phi\left(\frac{w-\mu}{\sigma}\right) \phi\left(\frac{v-\mu_{\gamma}}{\sigma_{\gamma}}\right) d v
$$

where $\mu$ is defined by:

$$
\mu=\beta_{0}+\beta_{1} \log \left[e^{x}-e^{v}\right]
$$

Figure 9 shows lines of equal probability of failure for different stress levels. In the analysis, the material fails if the simulated material capability is less than the stress level, including FOD effects, for $10^{6}$ cycles. The material capability is a random statistic that varies, but is correlated between blades on the same disk. This models that the blades all come from the same rough casting and would have similar capabilities.

\section{System reliability analysis}

The system analysis has multiple failure regimes since any node on any blade can exceed the material's stress capability. Traditional methods such as Fast Probability Integration (FPI) methods do not work with multiple failure zones. The number of random variables also rules against using complex analysis algorithms. Because of the reduced order models, simple random sampling (Monte Carlo Method) is acceptable to evaluate the system reliability. Using a standard desktop computer one million realizations can be run in a few hours. 
Table 1

Summary of random variables

\begin{tabular}{cccc}
\hline Variable & Distribution & Correlated & Number \\
\hline$a_{i}$ & Normal & No & $8 \times 24=192$ \\
Material & Uniform & Yes & $1 \times 24=24$ \\
FOD & Binomial & No & $10 \times 24=240$ \\
$K_{t}$ & Log-normal & No & $10 \times 24=240$ \\
\hline
\end{tabular}

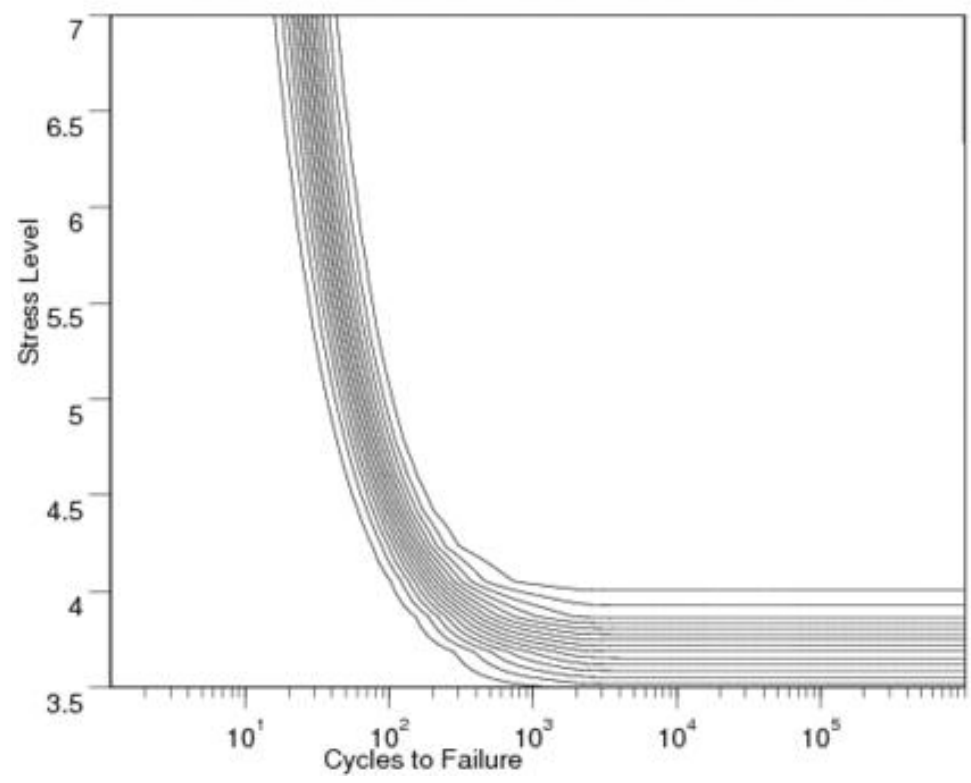

Fig. 9. Random fatigue limit model.

The high random variable count comes from the number of variables needed to define the blade geometry, material, and FOD. Each blade is fully defined by 29 random variables. When applied to a 24 blade rotor this translates to the 696 random variables shown in Table 1.

The blades are defined by their geometry and material properties. The PCA participation terms, $a_{i}$, each have their own standard deviation proportional to the height of the bar in Fig. 4. The material capability variables are correlated uniformly distributed variables. The rotor is milled from a single casting of metal and as such will have correlation of material capability from blade to blade although variation will still exist.

For the purposes of this model, the probability of FOD occurring on the leading edge increases linearly with the distance from the engine center-line. This is not exact but reflects the reality that majority of FOD is not found evenly distributed. The stress concentration distribution is randomly selected from the log-normal distribution.

The probabilistic integration was done through one million random realizations of rotors and FOD. It was determined that the failure probability is $0.0161 \pm 0.000247$. The convergence plot with confidence interval is shown in Fig. 10. The first failure rotor failure occurred on the $62^{\text {nd }}$ rotor as seen in the failure probability moving from 0.0. As more failed rotors are simulated the failure rate converges and the confidence interval decreases.

The confidence interval for the Monte Carlo analysis can be determined from the number of realizations $(n)$ and the number of failed realizations $\left(n_{f}\right)$. For a given confidence level $(\alpha)$ the probability that the true probability of failure $\left(p_{f}\right)$ is within one confidence interval of the estimate $\alpha$ as is shown in Eq. (12).

$$
P\left[p_{f} \in\left(\frac{n_{f}}{n} \pm t_{n-1, \frac{\alpha}{2}} \frac{\sqrt{\frac{n_{f}}{n}\left(1-\frac{n_{f}}{n}\right)}}{n}\right)\right]=\alpha
$$




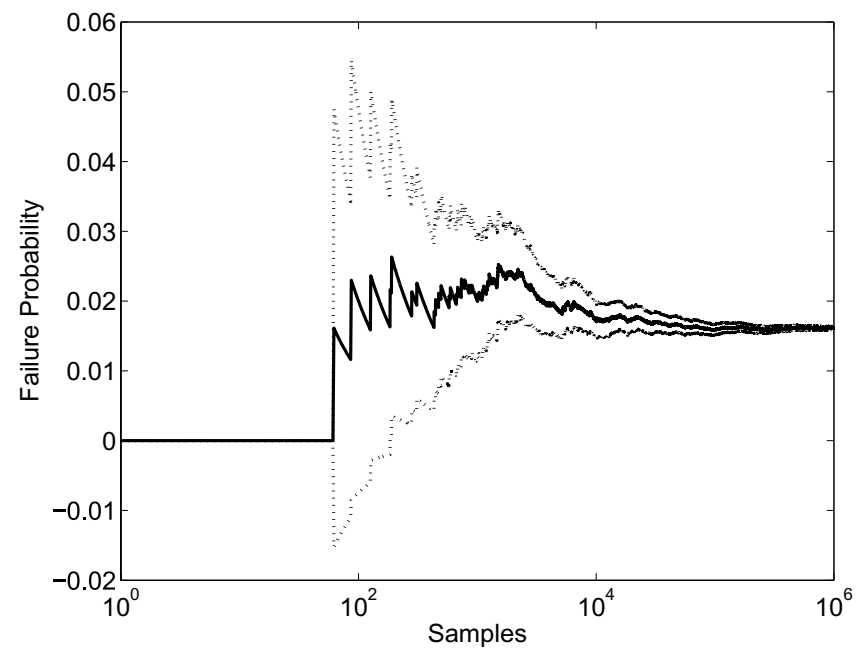

Fig. 10. Convergence of Monte Carlo analysis.

The confidence interval is important so that the error associated with the variability in the probabilistic method can be accounted for in the decision process after the analysis. Without the reported confidence interval the quality of the analysis may cause poor decisions to be made.

If the analysis would have been stopped after 1300 iterations, the failure rate estimate would have been 0.023 or almost $50 \%$ higher than the converged solution. However the confidence interval would have been 0.0082 meaning the true rate is likely to be anywhere between $1.5 \%$ or $3.1 \%$. Clearly that uncertainty range (plus or minus $35 \%$ ) is too large to base an engineering decision.

\section{Conclusions}

Reduced order models have made probabilistic implementation of the complete HCF design system shown in Fig. 1 possible. This study shows that it is tractable to connect the various models together to perform a probabilistic reliability assessment of an integrally bladed rotor. While questions remain about the input distributions, the integration error can and should be quantified. The differences in cost and reliability can feed the support planning of a new system.

Uncertainty analysis should be part of fielding a new design concept so that the proper decision analysis tools, such as value at risk or return on investment, can be used. Still the monetary benefits need to be compared with the performance increases that might not be evident in a standard cost benefit analysis.

The HCF design process would benefit significantly from accurate reduced order forced response or CFD code. This analysis could not account for variations in unsteady pressures or aerodynamic damping because the CFD models take too long to use within a Monte Carlo based analysis routine.

As the tools and computing speed increase a complete probabilistic HCF life analysis will be possible. The benefit over the current ad hoc approaches to using probabilistic methods within turbine engine design will be significant.

\section{References}

[1] Engine structural integrity program (ENSIP), Department of Defense Handbook MIL-HDBK-1783B, February 2002.

[2] J. Brown, J. Slater and R. Grandhi, Probabilistic analysis of geometric uncertainty on modal response, Proceedings of the 2003 IGTI Conference, no. GT2003-38557, June 2003.

[3] M.P. Castanier and C. Pierre, Modeling and analysis of mistuned bladed disk vibration: Status and emerging directions, Journal of Propulsion and Power 22 (2006)(2), 384-396.

[4] C.C. Chamis and A. Hamed, Probabilistic modeling for simulation of aerodynamic uncertainties in propulsion systems, Computing Systems in Engineering 1 (2-4) (1990), 373-390. 
[5] X. Chen, Foreign object damage on the leading edge of a thin blade, Mechanics of Materials 37 (2005), 447-457.

[6] B.M. Davis, S.R. Mannavat, T.J. Rockstroh and J.A. Hahn, Performance of GEN IV LSP for thick section airfoil damage tolerance, Proceedings of the 45th AIAA/ASME/ASCE/AHS/ASC Structures, Structural Dynamics and Materials Conference, no. AIAA 2004-2062, April 2004, pp. 5708-5717.

[7] Y.El-Aini, R. deLaneuville, A. Stoner and V. Capece, High cycle fatigue of turbomachinery components - industry perspective, Proceedings of the 33rd AIAA/ASME/SAE/ASEE Joint Propulsion Conference, no. AIAA-1997-3365, July 1997.

[8] D.J. Ewins, Vibration modes of mistuned bladed disks, ASME Journal of Engineering for Gas Turbines and Power 3(98) (1976), 349-355.

[9] Drew M. Feiner and Jerry H. GriEn, A fundamental model of mistuning for a single family of modes, Journal of Turbomachinery 124(4) (2002), 597-605.

[10] A. Muszynska and D.I.G. Jones, On tuned bladed disk dynamics: Some aspects of friction related mistuning, Journal of Sound and Vibration 1(86) (1983), 107-128.

[11] F.G. Pascual and W.Q. Meeker, Estimating fatigue curves with the random fatigue-limit model, Technometrics 41(4) (1999), $277-302$.

[12] J.O. Peters and R.O. Ritchie, Infuence of foreign-object damage on crack initiation and early crack growth during high-cycle fatigue of Ti-6Al-4V, Engineering Fracture Mechanics 67 (2000), 193-207.

[13] E.P. Petrov and D.J. Ewins, Eœects of damping and varying contact area at blade-disk joints in forced response analysis of bladed disk assemblies, Transactions of the ASME, The Journal of Turbomachinery 128(2) (2006), 403-410.

[14] P.S. Prevéy, N. Jayaraman, R.A. Ravindranath and M. Shepard, Improved high cycle fatigue damage tolerance of turbine-engine compressor components by low plasticity burnishing, J Eng Gas Turbines Power 130(1) (2008), 447-457.

[15] E.S. Reddy, G.H. Abumeri, P.L.N. Murthy and C.C. Chamis, Structural tailoring of aircraft engine blade subject to ice impact constraints, NASA Technical Memorandum 106033, September 1992, Prepared for Fourth AIAA/Air Force/NASA/OAI Symposium on Multidisciplinary Analysis.

[16] D.E. Thomson and J.T. GriEn, National turbine engine high cycle fatigue program, Global Gas Turbine News 39(1) (1999), $14-17$. 



Submit your manuscripts at

http://www.hindawi.com
